Анастасия В. Береснева, Анна В. Епишкина

\author{
Анастасия В. Береснева ${ }^{1}$, Анна В. Епишкина ${ }^{2}$ \\ 1,2 Национальный исследовательский ядерный университет «МИФИ», \\ Каширское ш., 31, Москва, 115522, Россия \\ Ie-mail: anastasiya3161@gmail.com,http://orcid.org/0000-0002-6214-6393 \\ ${ }^{2}$ e-mail: avepishkina@mephi.ru,http://orcid.org/0000-0001-7681-0382 \\ ПОДХОДЫ К ОНЛАЙН-ВЕРИФИКАЦИИ СОБСТВЕННОРУЧНОЙ ПОДПИСИ \\ DOI: http://dx.doi.org /10.26583/bit.2020.2.06
}

Аннотащия. Собственноручная подпись - один из наиболее распространенных методов биометрической аутентификации, где статические и динамические характеристики подписи используются для подтверждения личности пользователя. Целью исследования является анализ алгоритмов верификации собственноручной подписи и методов извлечения характеристик. Существующие разработки используют в основе различные технологии, такие как нейронная сеть, скрытая модель Маркова и алгоритмы машинного обучения. Помимо этого, на точность алгоритма верификации оказывает влияние метод извлечения характеристик подписи, а также, какие именно характеристики подписи принимаются во внимание в процессе классификации. Данная тематика бурно развивается, новые подходы и алгоритмы для решения задачи улучшают точность верификации и скорость обучения. По результатам исследования выбран алгоритм с наименьшим количеством ошибок первого и второго рода. Наиболее перспективный алгоритм положен в основу разрабатываемой системы аутентификации на основе собственноручной подписи.

Ключевые слова: верификачия, аутентификачия, биометрическая аутентификация, собственноручная подпись, машинное обучение, нейронная сеть.

Для иитирования: БЕРЕСНЕВА, Анастасия В.; ЕПИШКИНА, Анна В. ПОДХОДЫ К ОНЛАЙНВЕРИФИКАЦИИ СОБСТВЕННОРУЧНОЙ ПОДПИСИ. Безопасность информаџионных технологий, [S.l.], v. 27, n. 2, p. 78-85, 2020. ISSN 2074-7136. Доступно на: <https://bit.mephi.ru/index.php/bit/article/view/1272>. Дата docmyna: 27 may 2020. doi:http://dx.doi.org/10.26583/bit.2020.2.06.

\author{
Anastasia V. Beresneva ${ }^{1}$, Anna V. Epishkina ${ }^{2}$, \\ ${ }^{1,2}$ National Nuclear Research University MEPHI (Moscow Engineering Physics Institute), \\ Kashirskoe sh., 31, Moscow, 115522, Russia \\ le-mail: anastasiya3161@gmail.com, http://orcid.org/0000-0002-6214-6393 \\ ${ }^{2}$ e-mail: avepishkina@mephi.ru, http://orcid.org/0000-0001-7681-0382
}

\title{
Approaches to online handwritten signature verification
}

DOI: http://dx.doi.org /10.26583/bit.2020.2.06

Abstract. Handwritten signature is one of the most common methods of biometric authentication, where static and dynamic signature characteristics are used to confirm the user's identity. The existing developments are based on various technologies, such as the neural network, the hidden Markov model, and machine learning algorithms. This topic is rapidly developing, new approaches and algorithms for solving the problem improve the accuracy of verification and learning speed. The purpose of this study is to analyze existing approaches to the signature verification. The most promising algorithm will be used as the basis for the developed authentication system based on a handwritten signature.

Keywords: verification, authentication, biometric authentication, handwritten signature, machine learning, neural network.

For citation: BERESNEVA, Anastasia V.; EPISHKINA, Anna V. Approaches to online handwritten signature verification. IT Security (Russia), [S.l.], v. 27, n. 2, p. 78-85, 2020. ISSN 2074-7136. Available at: <https://bit.mephi.ru/index.php/bit/article/view/1272>. Date accessed: 27 may 2020. doi:http://dx.doi.org/10.26583/bit.2020.2.06.

\section{Введение}

Одной из приоритетных задач в области информационной безопасности является аутентификация и верификация личности пользователя. Система верификации - это 
совокупность методов, используемых с целью проверки правильности предоставленного идентификатора, сопоставляя его с эталонными образцами. Многие системы верификации основаны на биометрических технологиях, которые используют биологические характеристики человека для верификации.

Верификация собственноручной подписи - это биометрическая технология, которая использует подпись для идентификации личности с целью авторизации. Задача верификации подписи связана с определением того, действительно ли та или иная подпись принадлежит конкретному лицу или нет. Подписи особенно полезны для идентификации, так как подпись каждого человека уникальна, особенно если ее динамические характеристики рассматриваются вместе со статическим изображением. Алгоритмы, основанные на анализе динамических признаков, называются онлайн алгоритмами верификации подписи.

\section{1. Система верификации собственноручной подписи}

Основные этапы работы системы верификации собственноручной подписи включают в себя предварительную обработку, извлечение признаков и классификацию (рис. 1). В статье рассмотрены различные подходы к реализации данных этапов и проведено сравнение их эффективности.

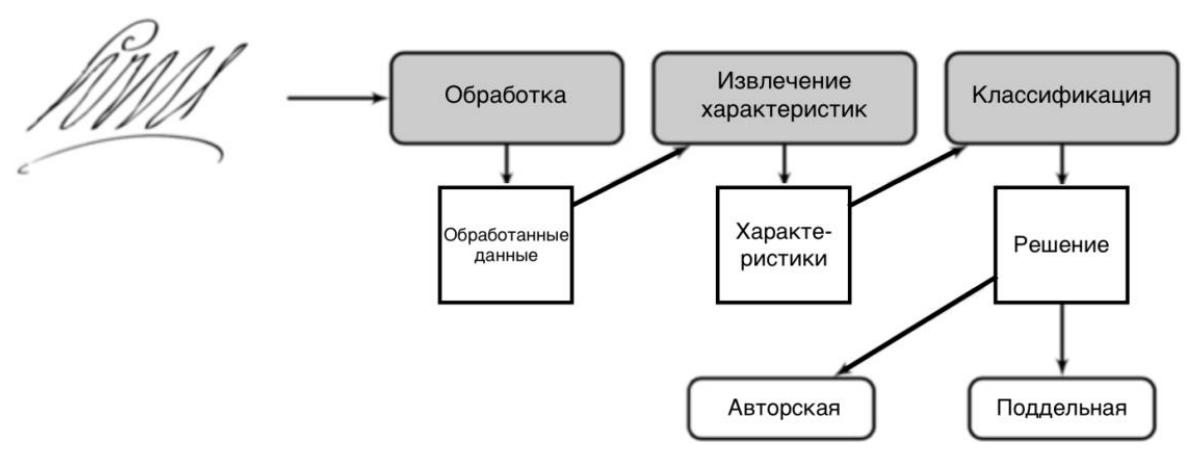

Рис.1. Этапь системы верификации собственноручной подписи

(Fig. 1. Stages of the handwritten signature verification system)

На первом этапе система верификации обрабатывает эталонный набор подписей автора. На основе данного набора происходит анализ и обучение классификатора [1].

После обучения следует этап оценки (тестирования). Во время оценки система верификации принимает на вход собственноручные подписи, чтобы определить, являются ли они подлинными или поддельными. Происходит сравнение эталонного набора с подписями, подлежащими верификации. Предварительная обработка, извлечение признаков одинаковы для подписей в эталонном и тестовом наборе, но эталонные наборы используются для задания характеристик метода (тонкой настройки параметров, например, увеличения или уменьшения порога и регулировки весов) и увеличения производительности [2].

На этапе классификации происходит принятие решения, является ли представленный образец оригинальным или поддельным относительно эталонного набора.

\section{1 Ввод подписи}

Собственноручная подпись для онлайн-верификации вводится на мобильном устройстве. Современные мобильные устройства обладают необходимым аппаратным обеспечением для извлечения динамических признаков собственноручной подписи, 
описывающих процесс совершения подписи - изменение координат пера в зависимости от времени, показания гироскопа и акселерометра.

\section{2 Обработка исходных данных}

Шаг предварительной обработки требуется перед извлечением признаков подписи главным образом для того, чтобы удалить шум. Предварительная обработка обычно включает фильтрацию, шумоподавление и сглаживание и часто выполняется с использованием преобразования Фурье [3, 4], гауссовых функций [5] или математической морфологии [6]. Нормализация подписей - это процесс приведения всех образцов к единому виду, который также является методом предварительной обработки. Обычно она включает смещение в сторону центра масс, который определяется как координата подписи, равноудаленная от краевых точек по вертикали и горизонтали, или начала координат [7]. В процессе обработки подпись разбивается на конечное число сегментов с приблизительно равным числом точек данных в каждом сегменте, таким образом, если подпись имеет Т точек и разделим ее на $\mathrm{S}$ сегментов, то сегменты будут иметь приблизительно $\mathrm{T} / \mathrm{S}$ точек в каждом сегменте. Сегментация сильно влияет на производительность верификации, поэтому хорошая техника сегментации может улучшить результаты верификации. Подпись разбивается на сегменты в местах резких перегибов, как приведено на рис. 2.
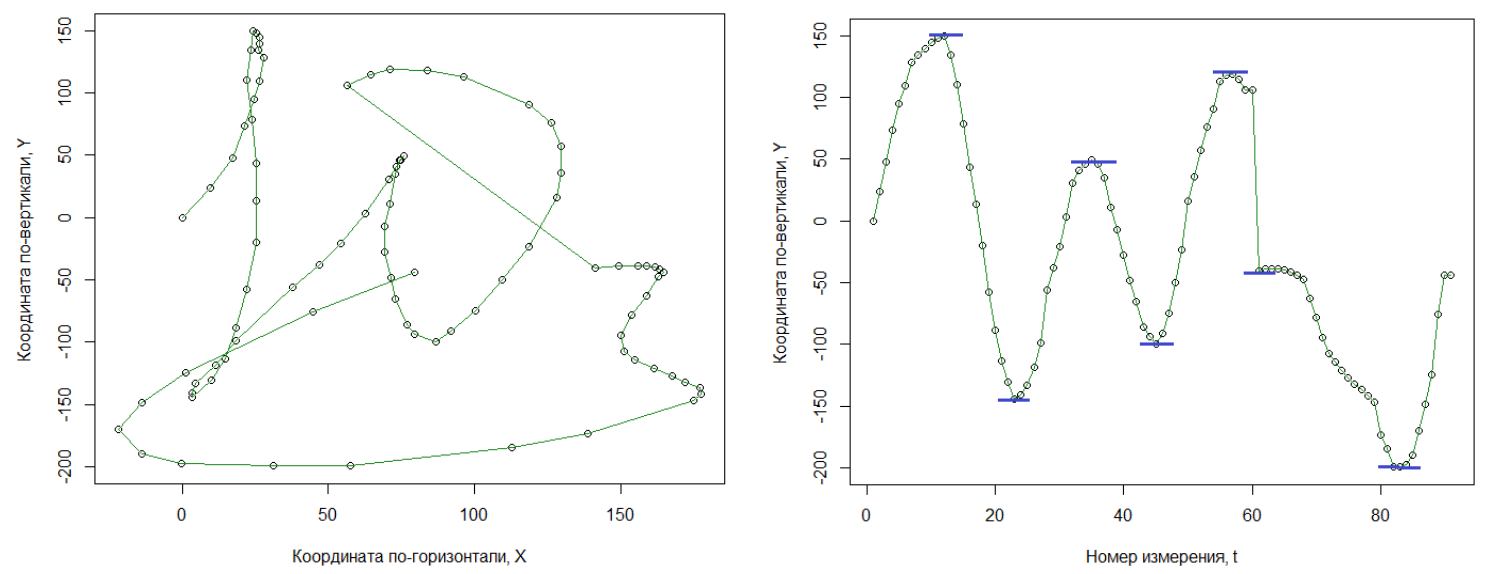

Рис. 2. Собственноручная подпись и зависимость у-координаты от времени

(Fig. 2. Handwritten signature and y-coordinate dependence on time)

\section{3 Извлечение характеристик}

Для того, чтобы определить, какие именно характеристики необходимо использовать в итоговом алгоритме для достижения максимальной точности верификации, необходимо провести исследование метода извлечения характеристик.

На данный момент различают три основных подхода к извлечению характеристик подписи [8]:

- Методы на основе локальных и глобальных параметров. Глобальные параметры извлекаются из всей подписи, а локальные - из ограниченной области подписи. В параметрических подходах для описания шаблона подписи выбирается набор параметров, затем параметры эталонных и тестовых подписей сравниваются, и выявляется подлинность подписи.

- Функциональные методы. Образец подписи представляется как функция времени, характеристики подписей локально сравниваются по принципу точка-точка или сегментсегмент [9]. В этом подходе динамические признаки подписи регистрируются как 
временные последовательности, содержащие информацию об изменениях во времени признаков подписи.

- Комбинированные (гибридные) методы. Этот подход основан на комбинировании разных методов из числа вышеприведенных [10].

В ходе исследования проанализированы различные методы выделения характеристик рукописной подписи:

- дискретное преобразование Фурье [3, 4];

- дискретное преобразование Радона [3];

- дискретное вейвлет-преобразование [5];

- извлечение характеристик подписи [2].

\section{4 Классификация}

В базовом сценарии при классификации подписи есть только несколько эталонных подписей от автора и тестируемая подпись. Цель состоит в том, чтобы решить, принадлежит ли тестируемая подпись автору, который совершил эталонные подписи, или нет. Этот сценарий называется классификацией одного класса. Для классификации могут быть использованы различные методы, рассмотренные ниже.

\subsection{1 Классификация на основе расстояний}

Данный метод классификации используется в сочетании с параметрическим методом извлечения признаков, в которых подпись представлена в виде набора векторов. Пусть $D$ - расстояние, которое проходит перо при написании собственноручной подписи. Иначе говоря, евклидово расстояние между всеми точками:

$$
D(Q, R)=\sqrt{\sum_{i=1}^{n}\left(q_{i}-r_{i}\right)^{2},}
$$

где $R=\left(r_{1}, r_{2}, \ldots, r_{n}\right)$ это характеристический вектор подписи автора, $Q=\left(q_{1}, q_{2}, \ldots, q_{n}\right)-$ характеристический вектор подписи, подлежащей классификации, $n-$ количество характеристик. Если евклидово расстояние меньше определенного порога, то подпись считается авторской.

\subsection{2 Алгоритм динамической трансформации временной шкалы}

Алгоритм позволяет оценить расстояние между двумя временными последовательностями разной длины, вычисляет матрицу преобразования и расстояние динамической трансформации [11]. На основании значения расстояния классификатор решает, является ли подпись подлинной или поддельной. Для выделения признаков при применении данного метода классификации используется функциональный метод извлечения признаков.

Таким образом, согласно алгоритму динамической трансформации, для двух векторов $u=\left(u_{1}, u_{2}, \ldots, u_{n}\right)$ и $v=\left(v_{1}, v_{2}, \ldots, v_{n}\right)$ расстояние трансформации может быть вычислено за $\mathrm{O}\left(\mathrm{n}^{2}\right)$. Матрица трансформации $C \in R^{((\mathrm{m}+1) \times(\mathrm{n}+1))}$ строится следующим образом:

$$
\begin{gathered}
C_{0,0}=0, C_{i, 0}=\infty, C_{0, j}=\infty ; i=1, \ldots n ; j=1, \ldots n ; \\
C_{i, j}=\left|u_{i}-v_{j}\right|+\min \left(C_{i-1, j}, C_{i, j-1}, C_{i-1, j-1}\right),
\end{gathered}
$$

где $\left|u_{i}-v_{j}\right|$ определяет абсолютное расстояние между координатой $i$ вектора $u$ и координатой $j$ вектора $v$. После построения матрицы $C$, можно вычислить наикратчайшее расстояние трансформации между векторами $u$ и $v$. 
Анастасия В. Береснева, Анна В. Епишкина

Алгоритм динамической трансформации временной шкалы был неоднократно улучшен в ряде исследований [12-13].

\subsection{3 Скрытая модель Маркова}

В этом методе классификации подпись может быть представлена в виде скрытой марковской модели (CMM), диаграмма перехода состояний которой приведена на рис. 3 . Согласно этой статистической модели, скрытая цепь переходит из состояния $i$ в состояние $i+1$ с вероятностью $a_{\mathrm{i}, \mathrm{i}+1}$ или возвращается в состояние $i$ с вероятностью $a_{i i}=1-a_{i, i+1}$. Пусть $q_{t}$ - состояние цепи в момент времени $t$; вероятность того, что вектор наблюдения $O_{t}$ внутри некоторой области $R_{j}$, когда цепь находится в состоянии $i$, определяется условной вероятностью состояния системы: $b_{i}(j)=P\left\{O_{t} \in R_{j} \mid q_{t}=i\right\}$.

В процессе обучения параметры исследуемой подписи оцениваются с помощью набора, содержащего эталонные подписи. Во время проверки вычисляется вероятность того, что подпись является подлинной. Если эта вероятность выше определенного порога, подпись принимается, в противном случае она отклоняется.

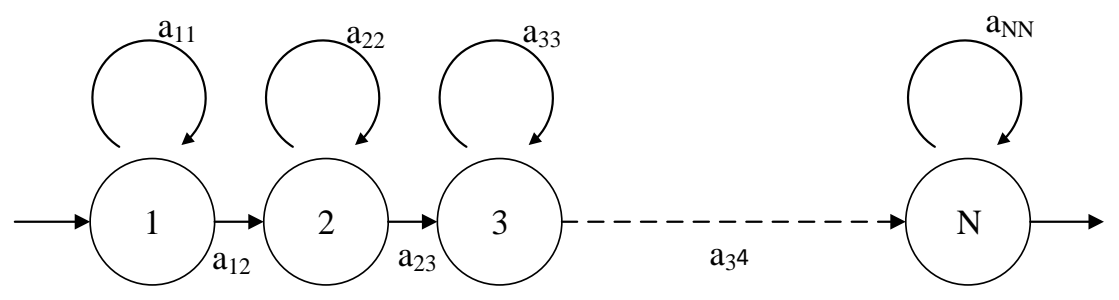

Рис.3. Диаграмма переходов состояний СММ

(Fig. 3. Diagram of HMM state transitions)

Этот подход можно рассматривать как статистическое соответствие между проверяемой подписью и подписью, основанной на СММ.

\subsection{5 Персептронная нейронная сеть}

Одним из методов классификации подписей является использование персептронной нейронной сети. Нейронная сеть - это система, состоящая из нейронов, их связей и ребер с определенными весами. Нейронная сеть получает вектор, содержащий значения характеристик подписи. Нейроны располагаются во входном и выходном слоях, а также в одном или нескольких скрытых слоях. Последний слой - это выход, который вычисляется суммированием и функцией активации.

В процессе обучения нейронная сеть учится корректировать веса ребер на основе известных классов данных обучения. Веса синапсов настраиваются таким образом, чтобы минимизировать ошибку - разницу между решенным выходом и выходом, вычисленным сетью на недавнем этапе на обучающем наборе. Применение нейронных сетей описывается в большом количестве исследований [14-15]. Для тестирования была реализована простая нейронная сеть персептрона, которая имеет 12 входов, два скрытых уровня по шесть нейронов в каждом и один выход.

\subsection{6 Метод опорных векторов}

Метод машинного обучения на основе опорных векторов классифицирует представленные образцы на основе обучающего набора данных. Основная идея метода состоит в том, чтобы перевести начальные векторы в пространство большей размерности и найти оптимальную гиперплоскость с максимальным зазором в этом пространстве. Этот 
классификационный подход используется в ряде работ [13-15]. Для извлечения признаков подписей могут быть использованы как функциональные, так и параметрические методы.

\section{2. Тестирование}

Для анализа эффективности указанных подходов к верификации собственноручных подписей были реализованы соответствующие алгоритмы. Кроме того, мобильное приложение для платформы Android на языке Java реализовано для сбора тестовых подписей пользователей и подписей злоумышленников.

Все реализованные алгоритмы основаны на предварительном обучении. Пользователь выполняет несколько подписей, из которых извлекаются необходимые характеристики.

Для оценки систем верификации используются коэффициенты ошибок 1-го и 2-го рода, которые называются коэффициентами ложного отклонения и ложного принятия соответственно:

- частота ложных пропусков - процент решений аутентификации, разрешающих доступ нелегитимному пользователю;

- частота ложных отказов - процент решений аутентификации, запрещающих доступ легитимному пользователю:

$$
F A R=\frac{N_{F A}}{N_{F}} \times 100 \%
$$

где $N_{F A}$ и $N_{F R}$ - число ложно принятых и число ложно отклоненных собственноручных подписей на тестовом наборе, $N_{F}$ - число поддельных подписей, $N_{T}$ - число оригинальных подписей [5].

По результатам тестирования на наборе из 100 подписей для рассматриваемых алгоритмов получены результаты, приведенные в табл. 1. Для тестирования метода опорных векторов также сформирован набор из подписей злоумышленника. Для формирования данного набора пользователям предлагалось проследить за совершением подписи автором, а затем повторить подпись от лица злоумышленника.

Исследование показало, что наиболее перспективными методами для дальнейшей работы являются алгоритмы на основе нейронной сети и метода опорных векторов, поскольку доля ошибок 1-го и 2-го рода для этих алгоритмов минимальна относительно других. Существует несколько причин возникновения ошибок 1-го рода. Во-первых, некоторые люди не могут приспособиться к подписи на планшете, а подлинные подписи на планшете отличаются от подписей на бумаге. Во-вторых, набор эталонных подписей исходного пользователя не является достаточным для обучения этим методам.

Таблища 1. Результаты тестирования реализованных алгоритмов

\begin{tabular}{|c|c|c|c|c|c|c|c|c|}
\hline Метод & \multicolumn{2}{|c|}{$\begin{array}{c}\text { Дискретное } \\
\text { преобразование } \\
\text { Фурье }\end{array}$} & \multicolumn{2}{c|}{$\begin{array}{c}\text { Дискретное } \\
\text { преобразование } \\
\text { Радона }\end{array}$} & \multicolumn{2}{|c|}{$\begin{array}{c}\text { Вейвлет } \\
\text { преобразование }\end{array}$} & \multicolumn{2}{|c|}{ Характеристики } \\
\hline $\begin{array}{c}\text { Алгоритм } \\
\text { на основе } \\
\text { расстояний }\end{array}$ & $19 \%$ & $17 \%$ & $15 \%$ & $8 \%$ & $13 \%$ & $4 \%$ & $11 \%$ & $14 \%$ \\
\hline $\begin{array}{c}\text { Алгоритм } \\
\text { динамической } \\
\text { трансформации }\end{array}$ & $11 \%$ & $18 \%$ & $14 \%$ & $10 \%$ & $10 \%$ & $8 \%$ & $10 \%$ & $12 \%$ \\
\hline $\begin{array}{c}\text { Скрытая модель } \\
\text { Маркова }\end{array}$ & $10 \%$ & $17 \%$ & $17 \%$ & $7 \%$ & $14 \%$ & $17 \%$ & $8 \%$ & $7 \%$ \\
\hline
\end{tabular}


Анастасия В. Береснева, Анна В. Епишкина

ПОДХОДЫ К ОНЛАЙН-ВЕРИФИКАЦИИ СОБСТВЕННОРУЧНОЙ ПОДПИСИ

\begin{tabular}{|c|c|c|c|c|c|c|c|c|}
\hline Нейронная сеть & $9 \%$ & $12 \%$ & $12 \%$ & $17 \%$ & $13 \%$ & $19 \%$ & $12 \%$ & $7 \%$ \\
\hline $\begin{array}{c}\text { Метод опорных } \\
\text { векторов }\end{array}$ & $9 \%$ & $9 \%$ & $13 \%$ & $7 \%$ & $9 \%$ & $12 \%$ & $10 \%$ & $7 \%$ \\
\hline & $\begin{array}{c}\text { Ошибки } \\
\text { 1-го рода }\end{array}$ & $\begin{array}{c}\text { Ошибки } \\
\text { 2-го рода }\end{array}$ & $\begin{array}{c}\text { Ошибки } \\
\text { 1-го рода }\end{array}$ & $\begin{array}{c}\text { Ошибки } \\
\text { 2-го рода }\end{array}$ & $\begin{array}{c}\text { Ошибки } \\
\text { 1-го рода }\end{array}$ & $\begin{array}{c}\text { Ошибки } \\
\text { 2-го рода }\end{array}$ & $\begin{array}{c}\text { Ошибки } \\
\text { 1-го рода }\end{array}$ & \begin{tabular}{c} 
2шибо рода \\
\hline
\end{tabular} \\
\hline
\end{tabular}

\section{Заключение}

Рассмотренные алгоритмы верификации подписи имеют достаточно высокие показатели эффективности, однако доля ошибок может быть сокращена в результате усовершенствования метода извлечения признаков подписи в дальнейшем исследовании.

По результатам исследования, приведенным в таблице, можно заметить, что функциональные методы извлечения характеристик дают худший результат в сравнении с локальным методом извлечения. При использовании локальных методов извлечения характеристик подписи значения ошибок первого и второго рода минимальны, а значит точность алгоритма верификации выше. На основе этого можно сделать вывод, что статические и динамические характеристики подписи, извлеченные на каждом интервале, позволяют подробнее описать процесс совершения подписи. В то же время не все характеристики являются информативными, многие из них дублируются либо не являются устойчивыми по времени. Для анализа значимости характеристик в дальнейшем исследовании необходимо провести анализ на основе статистических свойств.

Помимо этого, в дальнейшей работе планируется разработка алгоритма верификации собственноручной подписи на основе алгоритмов машинного обучения, основанного на локальных характеристиках с целью сокращения ошибок первого и второго рода, а также времени обучения и принятия решения.

\section{СПИСОК ЛИТЕРАТУРЫ:}

1. Ooi, Shih Yin, Andrew Beng Jin Teoh, Ying-Han Pang and Bee Yan Hiew. "Image-based handwritten signature verification using hybrid methods of discrete Radon transform, principal component analysis and probabilistic neural network." Appl. Soft Comput. Vol. 40, $2016 . \quad$ P. 274-282. DOI: https://doi.org/10.1016/j.asoc.2015.11.039.

2. Wróbel, Krzysztof \& Doroz, Rafal \& Porwik, Piotr \& Naruniec, Jacek \& Kowalski, Marek. (2017). Using a Probabilistic Neural Network for lip-based biometric verification. Engineering Applications of Artificial Intelligence. 64. 112-127. DOI: https://doi.org/10.1016/j.engappai.2017.06.003.

3. Buchegger, P. Hacking Fingerprint Readers Without Making A Mess. Syss. URL:https://www.syss.de/fileadmin/dokumente/Publikationen/2018/Hacking_Fingerprint_Readers_without_M aking_a_Mess.pdf (дата обращения: 25.12.2019).

4. Deng, Y. Keystroke Dynamics Advances for Mobile Devices Using Deep Neural Network Y. Deng, Y. Zhong. Recent Advances in User Authentication Using Keystroke Dynamics Biometrics. 2015. Vol. 2. P. 59-70. DOI: https://doi.org/10.15579/gcsr.vol2.ch4.

5. Rosso OA, Ospina R, Frery AC. Classification and Verification of Handwritten Signatures with Time Causal Information Theory Quantifiers // Journal of Computer Aided Design and Computer Graphics. Vol. 19, No. 3. P.243-301. DOI: https://doi.org/10.1371/journal.pone.0166868.

6. L. Lu and Y. Liu, "Safeguard: User Reauthentication on Smartphones via Behavioral Biometrics," in IEEE Transactions on Computational Social Systems, Vol. 2, No. 3. P. 53-64, Sept. 2015. DOI: https://doi.org/10.1109/TCSS.2016.2517648.

7. L. Zhang, L. Zhang and B. Du, "Deep Learning for Remote Sensing Data: A Technical Tutorial on the State of the Art," in IEEE Geoscience and Remote Sensing Magazine, Vol. 4, No. 2. P. 22-40, June 2016. DOI: https://doi.org/10.1109/MGRS.2016.2540798.

8. Al-Ani, Muzhir. (2015). Signature Recognition Using Discrete Fourier Transform. DOI: https://doi.org/10.13140/RG.2.1.5182.6645.

9. Gao ZK, Yang Y-X, Zhai L-S, Ding M-S, Jin N-D. Characterizing slug to churn flow transition by using multivariate pseudo Wigner distribution and multivariate multiscale entropy. Chemical Engineering Journal. Vol. 291, 2016. P. 74-81. DOI: https://doi.org/10.1016/j.cej.2016.01.039.

10. Rosso, Osvaldo \& Olivares, Felipe \& Plastino, A. (2015). Noise versus chaos in a causal Fisher-Shannon plane. 1852-4249. 7. DOI: https://doi.org/10.4279/PIP.070006. 
Анастасия В. Береснева, Анна В. Епишкина

11. Jain, A., Singh, S.K. \& Singh, K.P. Handwritten signature verification using shallow convolutional neural network. Multimed Tools Appl (2020). DOI: https://doi.org/10.1007/s11042-020-08728-6.

12. Yalin Yin and Xiangdong Zhou "End-to-end online handwriting signature verification", Proc. SPIE 11069, Tenth International Conference on Graphics and Image Processing (ICGIP 2018), 1106921 (6 May 2019). DOI: https://doi.org/10.1117/12.2524447.

13. Alona Levy, Ben Nassi, Yuval Elovici, and Erez Shmueli. 2018. Handwritten Signature Verification Using Wrist-Worn Devices. Proc. ACM Interact. Mob. Wearable Ubiquitous Technol. 2, 3, Article 119 (September 2018). - 26 p. DOI:https://doi.org/10.1145/3264929.

14. Beltramelli, T., \& Risi, S. (2015). Deep-Spying: Spying using Smartwatch and Deep Learning. ArXiv, abs/1512.05616.

15. Kumar, R., Phoha, V.V., \& Raina, R. (2016). Authenticating users through their arm movement patterns. ArXiv, abs/1603.02211.

\section{REFERENCES:}

[1] Ooi, Shih Yin, Andrew Beng Jin Teoh, Ying-Han Pang and Bee Yan Hiew. "Image-based handwritten signature verification using hybrid methods of discrete Radon transform, principal component analysis and probabilistic neural network." Appl. Soft Comput. Vol. 40, $2016 . \quad$ P. 274-282. DOI: https://doi.org/10.1016/j.asoc.2015.11.039.

[2] Wróbel, Krzysztof \& Doroz, Rafal \& Porwik, Piotr \& Naruniec, Jacek \& Kowalski, Marek. (2017). Using a Probabilistic Neural Network for lip-based biometric verification. Engineering Applications of Artificial Intelligence. 64. 112-127. DOI: https://doi.org/10.1016/j.engappai.2017.06.003.

[3] Buchegger, P. Hacking Fingerprint Readers Without Making A Mess. Syss.

URL:https://www.syss.de/fileadmin/dokumente/Publikationen/2018/Hacking_Fingerprint_Readers_without_M aking_a_Mess.pdf (accessed: 25.12.2019).

[4] Deng, Y. Keystroke Dynamics Advances for Mobile Devices Using Deep Neural Network Y. Deng, Y. Zhong. Recent Advances in User Authentication Using Keystroke Dynamics Biometrics. 2015. Vol. 2. P. 59-70. DOI: https://doi.org/10.15579/gcsr.vol2.ch4.

[5] Rosso OA, Ospina R, Frery AC. Classification and Verification of Handwritten Signatures with Time Causal Information Theory Quantifiers. Journal of Computer Aided Design and Computer Graphics. Vol. 19, No. 3. P.243-301. DOI: https://doi.org/10.1371/journal.pone.0166868.

[6] L. Lu and Y. Liu, "Safeguard: User Reauthentication on Smartphones via Behavioral Biometrics," in IEEE Transactions on Computational Social Systems, Vol. 2, No. 3. P. 53-64, Sept. 2015. DOI: https://doi.org/10.1109/TCSS.2016.2517648.

[7] L. Zhang, L. Zhang and B. Du, "Deep Learning for Remote Sensing Data: A Technical Tutorial on the State of the Art," in IEEE Geoscience and Remote Sensing Magazine, Vol. 4, No. 2. P. 22-40, June 2016. DOI: https://doi.org/10.1109/MGRS.2016.2540798.

[8] Al-Ani, Muzhir. (2015). Signature Recognition Using Discrete Fourier Transform. DOI: https://doi.org/10.13140/RG.2.1.5182.6645.

[9] Gao ZK, Yang Y-X, Zhai L-S, Ding M-S, Jin N-D. Characterizing slug to churn flow transition by using multivariate pseudo Wigner distribution and multivariate multiscale entropy. Chemical Engineering Journal. Vol. 291, 2016. P. 74-81. DOI: https://doi.org/10.1016/j.cej.2016.01.039.

[10] Rosso, Osvaldo \& Olivares, Felipe \& Plastino, A. (2015). Noise versus chaos in a causal Fisher-Shannon plane. 1852-4249. 7. DOI: https://doi.org/10.4279/PIP.070006.

[11] Jain, A., Singh, S.K. \& Singh, K.P. Handwritten signature verification using shallow convolutional neural network. Multimed Tools Appl (2020). DOI: https://doi.org/10.1007/s11042-020-08728-6.

[12] Yalin Yin and Xiangdong Zhou "End-to-end online handwriting signature verification", Proc. SPIE 11069, Tenth International Conference on Graphics and Image Processing (ICGIP 2018), 1106921 (6 May 2019). DOI: https://doi.org/10.1117/12.2524447.

[13] Alona Levy, Ben Nassi, Yuval Elovici, and Erez Shmueli. 2018. Handwritten Signature Verification Using Wrist-Worn Devices. Proc. ACM Interact. Mob. Wearable Ubiquitous Technol. 2, 3, Article 119 (September 2018). - 26 p. DOI:https://doi.org/10.1145/3264929.

[14] Beltramelli, T., \& Risi, S. (2015). Deep-Spying: Spying using Smartwatch and Deep Learning. ArXiv, abs/1512.05616.

[15] Kumar, R., Phoha, V.V., \& Raina, R. (2016). Authenticating users through their arm movement patterns. ArXiv, abs/1603.02211.

Поступила в редакцию - О9апреля 2020 г. Окончательньий вариант - 25 мая 2020 г. Received-April 09, 2020. The final version-May 25, 2020. 